\title{
Illumination invariant face recognition using dual-tree complex wavelet transform in logarithm domain
}

\author{
Guang Yi Chen, Tien D. Bui, Adam Krzyzak*
}

\begin{abstract}
In this article, we develop a new algorithm for illumination invariant face recognition. We first transform the face images to the logarithm domain, which makes the dark regions brighter. We then use dual-tree complex wavelet transform to generate face images that are approximately invariant to illumination changes and use collaborative representation-based classifier to classify the unknown faces to one known class. We set the approximation sub-band and the highest two DTCWT coefficient sub-bands to zero values before the inverse DTCWT transform is performed. Experimental results demonstrate that our proposed method improves upon a few existing methods under both the noise-free and noisy environments for the Extended Yale Face Database B and the CMU-PIE face database.

Keywords: face recognition, invariant features, dual-tree complex wavelet transform (DTCWT), collaborative representation-based classifier (CRC)
\end{abstract}

\section{Introduction}

Face recognition is a popular research topic in such diverse applications as military, commercial, and public security. A huge variety of variations exist in human faces because of illumination, shift, pose, occlusion, and expression. There are three categories of methods to deal with illumination variation in face recognition. The first category of methods extracts illumination invariant features from the faces. The second category of methods creates a $3 \mathrm{D}$ shape model to deal with illumination difference in human faces. The third category of methods utilizes image-processing techniques to normalize different illumination conditions. In this paper, we choose the third category of method for invariant face recognition because it achieves good classification results as demonstrated in the experiments in this paper.

We discuss several existing face recognition algorithms here. Eigenfaces $[1,2]$ are a very important method for face recognition, which calculates the principal components of the human face images. The eigenfaces build a mathematical model, which best describes a face by extracting the most relevant information contained in that face. Laplacian faces [3] are superior to eigenfaces for face recognition, where the face images are analysed by using locality-preserving projections. Lee et al [4] studied face recognition under variable lighting conditions. In their paper, they arranged physical lighting in such a way that the acquired images of each object can be directly used as the basis vectors of a low-dimensional linear space. Luu et al [5] studied multifactor analysis for face recognition with adaptive factor structures. They proposed Compressed Submanifold Multifactor Analysis (CSMA) and their method can preserve the original local geometry structures of input factors. Yang et al [6] proposed a novel wavelet-based method for face recognition. In their paper, they demonstrated that nonlinear approximation preserves more information in an image than linear approximation. Wright et al [7] studied robust face recognition via sparse representation. They studied the problem of automatically recognizing human faces from frontal views with varying expression, illumination, occlusion, and disguise. They treated the recognition problem as classifying among multiple linear regression models, and their new theory from sparse signal representation offers the key to addressing this problem. Du and Ward [8] proposed a region-based image enhancement method for face recognition. Nevertheless, the method yields defects on the boundary between different image regions. Ruiz-Pinales et al [9] invented a translation-invariant support vector machines (SVM) for facial image recognition. In their article, they presented a new technique for incorporating global translation invariance in SVM. While other methods incorporate a feature extraction stage, they scaled the image and then classified it by using the modified SVM classifier. Translation invariance is achieved by replacing dot products between patterns with the maximum cross-correlation value. Chen et al [10] studied illumination normalization for robust face recognition by first performing logarithm transform to the face images and then conducting discrete cosine transform (DCT) to the logarithm images (LOG-DCT). In this method, they set the low-resolution DCT coefficients to zero and then perform inverse DCT in order

* Department of Computer Science and Software Engineering, Concordia University, Montreal, Quebec, Canada H3G 1M8, guangyi_chen@hotmail.com 


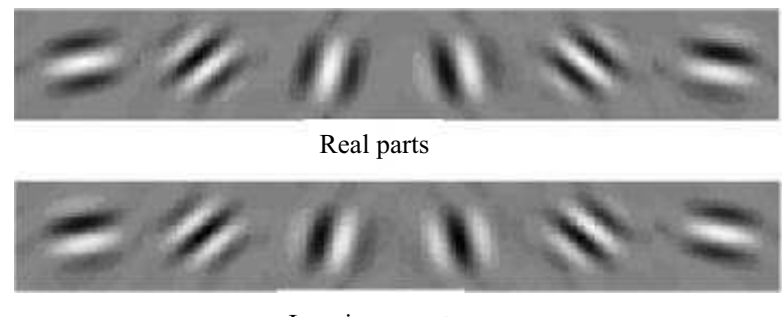

Imaginary parts

Fig. 1. The 2D DTCWT transform with six dominant orientations

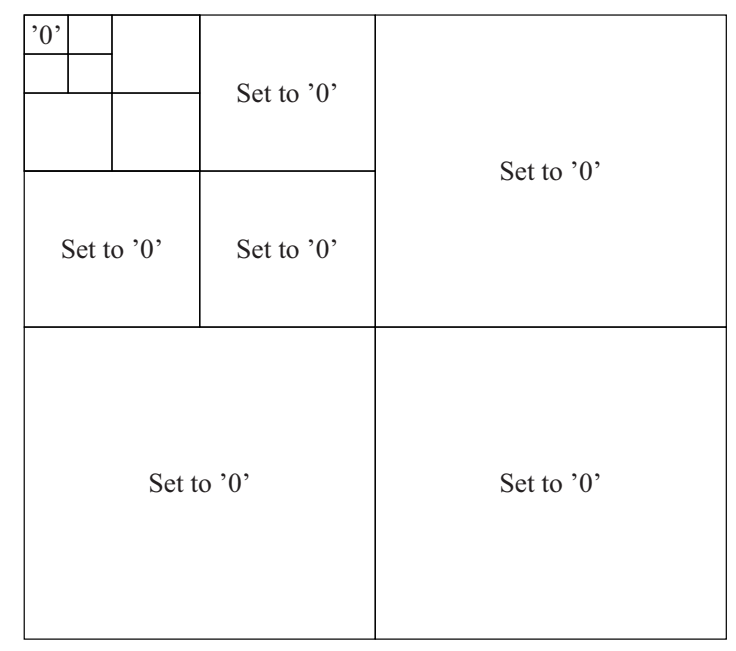

Fig. 2. An illustration of setting the approximation and the two highest frequency subbands to zero

to obtain illumination invariant face maps for classification. Chen et al [11] proposed a log total variation model (LTV) for face recognition under variable lighting conditions. However, it is relatively complex than other methods because it needs to solve differential equations. Ahonen et al [12] studied face description and recognition with local binary patterns (LBP). They presented a new and fast face image representation based on LBP texture features. They divided the face images into several regions, extracted the LBP feature distributions, and then concatenated into a feature vector as a face descriptor. Liu and Dai [13] worked on face recognition using dualtree complex wavelet transform (DTCWT) features. This method extracts features from the face images and uses these features to classify unknown face to one existing face class. Zhang et al [14] investigated face recognition by means of gradient faces under varying illumination conditions. However, these gradient faces are very sensitive to noise. Lai et al [15] recently developed a face recognition method using multiscale logarithm difference edgemaps under varying lighting conditions. In this method, they utilized a logarithm difference model, which eliminates light intensity from pixels in a neighbourhood. This method is better than holistic image decomposition methods such as LOG-DCT and LTV. Compared with Gradient faces and Weber faces, there exist two advantages for this method. First, logarithm transform has a physi- ological basis and a good mathematical property, which is better than ill-posed quotient models. Second, it extracts edge maps of different scales with respect to different sub-regions in a neighborhood, but Gradient faces and Weber faces only involve a few neighboring pixels that are not sufficient for multi-scale treatments. Shah et al [16] proposed a robust face recognition technique under varying illumination conditions. They resolved the limitations of existing methods by transforming pixels from non-illuminated side to illuminated side. They reported that their proposed method produced better results than other existing methods compared in their paper such as self-quotient image (SQI) and multi-scale retinex (MSR), etc.

We briefly review several face recognition algorithms that used DTCWT here. Liu and Dai [13] extracted DTCWT features for illumination invariant face recognition. However, our new method is different from [13] because we not only set the highest two DTCWT coefficient sub-bands to zero values but also set the approximation subband to zero. Furthermore, we perform inverse DTCWT to obtain illumination invariant faces whereas [13] works in the feature space. Selesnick et al [17] also developed a novel method for face recognition due to the attractive properties of the DTCWT: approximate shift-invariance, orientation selectivity, and efficient computation. Zhang et al [18] performed one level DTCWT to the face image and then used SVM as a classifier for face recognition. In [19] and [20], the authors performed the DTCWT transform for four decomposition scales, and the high-frequency sub-bands are employed to construct the representation of faces. Nevertheless, they simply utilized DTCWT and did not study the performance of DTCWT for face representation under different variations such as shift and illumination. Principal component analysis (PCA) was used for dimension reduction, but it was not clear how to choose the number of principal components to retain.

In this article, we propose a novel method for face recognition by means of DTCWT [21] to generate illumination invariant face images, and by using collaborative representation-based classifier (CRC) to classify the faces. Our method improves upon a few existing methods for both the Extended Yale Face Database B and the CMU-PIE illumination face database. Furthermore, our proposed method does not require any modelling. In addition, they can be applied directly to any face image without any lighting assumption or any prior information on $3 \mathrm{D}$ face geometry.

\section{Methodology}

During the last two decades, face recognition has become a very important research topic in computer vision, image analysis, and understanding. Due to illumination differences, the acquired face images may be very dark locally or globally. Therefore, existing face recognition methods will have lower recognition rates when the lighting conditions are not ideal [10]. 


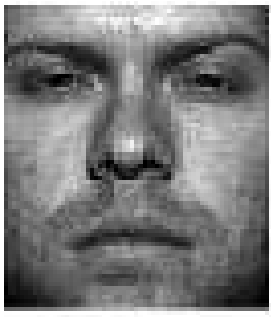

(a)

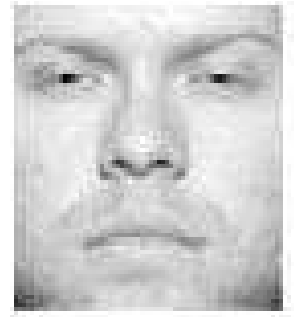

(b)

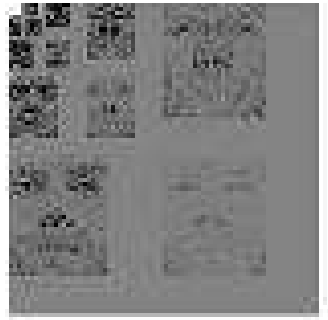

(c)

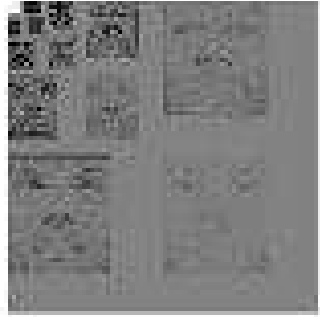

(d)

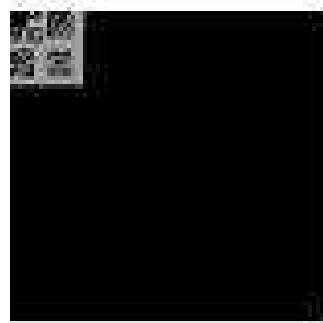

(e)

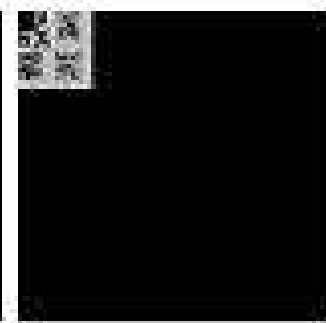

(f)

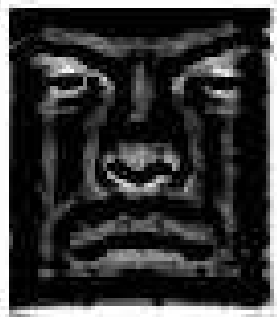

(g)

Fig. 3. The different stages of our illumination invariant face recognition algorithm: (a) - the input face image, (b) - the logarithm of the input image, (c) - tree 1 of the DTCWT transform on the logarithm image, (d) - tree 2 of the DTCWT transform on the logarithm image, (e) - set the approximation and the two highest frequency scales of tree 1 to zero, (f) - set the approximation and the two highest frequency scales of tree 2 to zero, $(\mathrm{g})$ - the output illumination invariant face generated by inverse DTCWT

In the following, we will briefly discuss the Lambertian reflectance theory, the DTCWT transform, and the CRC classifier. According to the Lambertian reflectance theory [22], the intensity image can be modeled as

$$
I(x, y)=R(x, y) L(x, y)
$$

where, $R$ is the reflectance and $L$ is the illumination. Because $R$ depends only on the surface material of the subject, it is the intrinsic representation of a face image. The more suitable solution is to convert the intensity image of the face to the logarithm domain, where the multiplication becomes addition

$$
\log I(x, y)=\log R(x, y)+\log (L(x, y)
$$

making the face recognition easier.

The DTCWT transform [21] developed by Kingsbury is known to be approximately shift-invariance, and it is better than standard discrete wavelet transform (DWT). This transform overcomes the shift-variance problem of the decimated DWT and it involves two parallel DWT channels with the corresponding wavelets. The DTCWT transform has six directionally selective filters (See Fig. 1 ), while standard wavelet transform has only two dominant orientations. The DWT is very sensitive to spatial shifts: a little shift in spatial domain will cause very different wavelet coefficients [21]. This is the main reason why we choose DTCWT in this paper.

Face recognition is not a trivial job due to large illumination variations. Nevertheless, illumination does not change as quickly as the reflectance in an image. As a result, illumination variations are mainly in the low frequency sub-bands. Since we want to recognize faces using reflectance, illumination variations can be reduced by removing low-frequency sub-bands. In addition, the highest frequency sub-bands mostly contain noise, so removing the highest frequency sub-bands is also desirable in face recognition. This is because the highest frequency subbands may contain a lot of noise and so they are very unstable for face recognition.

In the last decade, sparse representation-based classification (SRC) [7] is very popular in robust face recognition. This is because the SRC represents an unknown face image as a sparse linear combination of its training face images. It recognizes the face image by calculating the distance between the faces and the class with the minimal error is assigned the true class of the unknown face image. Even though the $l_{1}$-norm sparsity constraint on coding coefficients plays an important role in the success of SRC, the training samples that collaboratively represent the query image is usually not studied. Zhang et al [23] pointed out that the collaborative representation scheme in SRC is more important to the success of face classification. The SRC is a special case of collaborative representation-based classification (CRC), and it has many instantiations by using other norms to the coding residual and coding coefficient. As a result, we will take advantage of this CRC for our face classification task. In $\mathrm{CRC}$, one needs to solve the following optimization problem

$$
\tilde{a}_{k}=\arg \min _{\alpha}\left\|b_{k}-A \alpha\right\|_{2}^{2}+\lambda\|\alpha\|_{2}^{2}
$$

where $\lambda$ is a parameter and $\tilde{\alpha}_{k}=\left[\tilde{\alpha}_{k 1} \tilde{\alpha}_{k 2} \cdots \tilde{\alpha}_{k C}\right]$. This optimization problem has a closed-form solution with $k \in$ $[1, M]$.

$$
\tilde{\alpha}_{k}\left(A^{\top} A+\lambda I\right)^{-1} A^{\top} b_{k}
$$

where the $A^{\top}$ means the transpose of the matrix $A$. Because $D=\left(A^{\top} A+\lambda I\right)^{-1} A^{\top}$ can be computed offline, it is fast to calculate $\tilde{\alpha}_{k}=D b_{k}$. Assume $e_{k i}=\| b_{k}-$ 


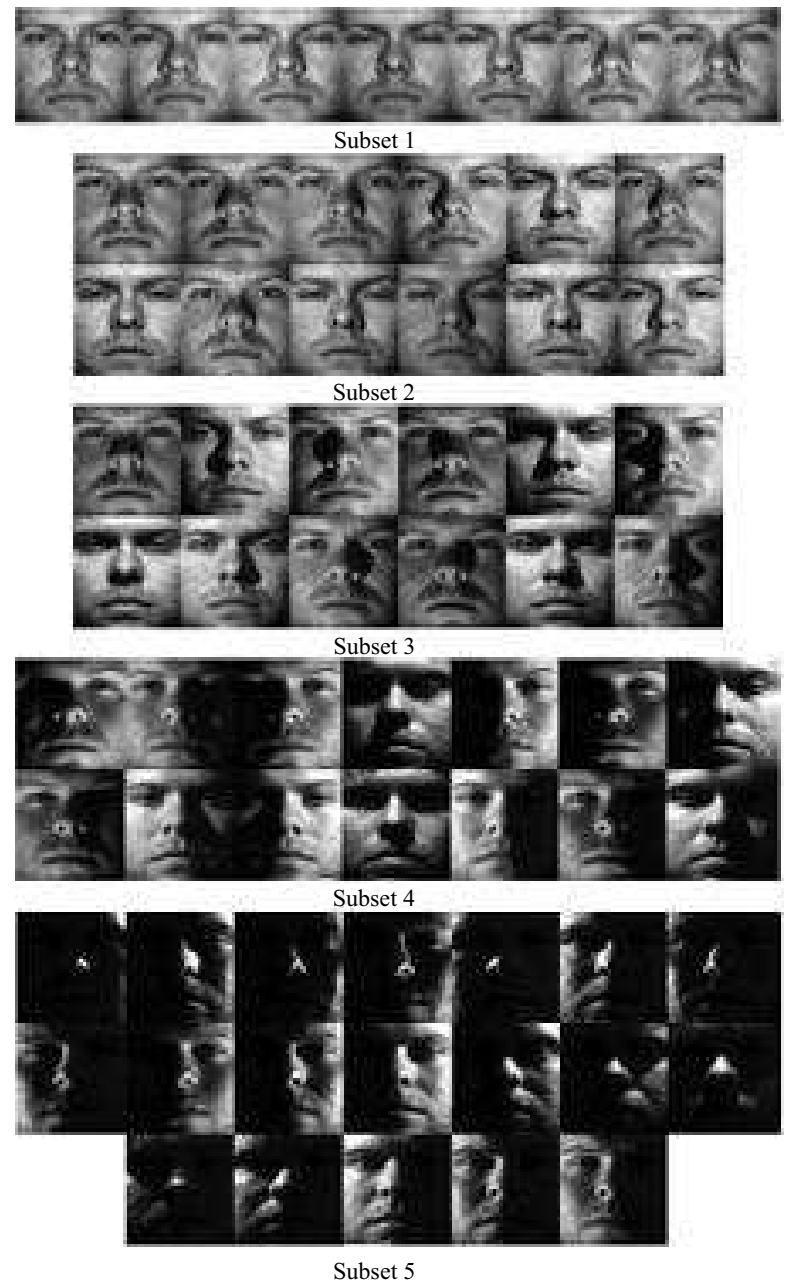

Fig. 4. The five subsets of the Extended Yale-B face database

$A_{i} \tilde{\alpha}_{k i} \|_{2}$ and $e_{k}=\left(e_{k 1} e_{k 2} \cdots e_{k C}\right)^{\top}$. The CRC classifies a face $b_{k}$ as $z_{k}=$ identity $b_{k}=\arg \min _{i}\left\{e_{k i}\right\}$. The CRC classification can be achieved by checking which class produces the smallest reconstruction error. The speed of the $\mathrm{CRC}$ is much faster than the $\mathrm{SRC}$ for face recognition.

Inspired by LOG-DCT [10], in this article, we develop a new method for illumination invariant face recognition. Because of different illumination conditions, the acquired facial images may be very dark, which makes existing face recognition methods fail. In order to improve classification rates, we propose to elevate the dark region and suppress bright region by means of logarithm transform. We perform DTCWT transform to these logarithm images and set the approximation sub-band and the two highest frequency DTCWT sub-bands to zero values (see Fig. 2). An inverse DTCWT transform will generate our enhanced face images, which are approximately invariant to illumination. The reason why we choose DTCWT is because this transform has approximate shift invariant property, which is very successful in our previous works for character recognition [24], signal denoising [25], and shape recognition [26].

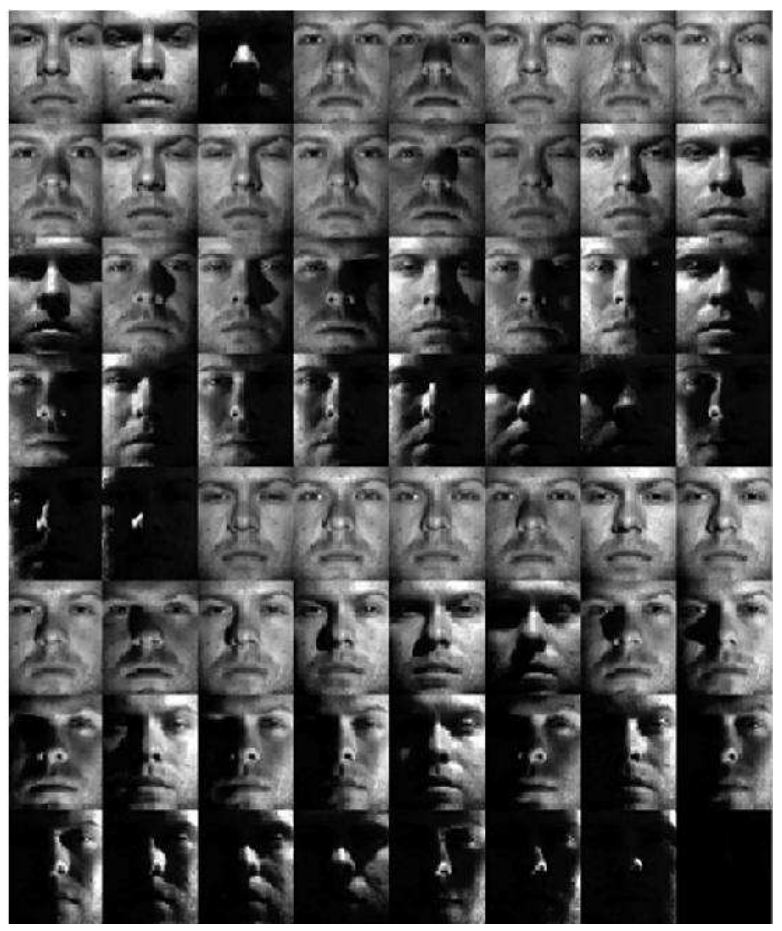

Fig. 5. A sample of the face images under different lighting condition of the Extended Yale-B face database

Figure 3 shows the different stages of our illumination invariant face recognition algorithm: (a) the input face image, (b) the logarithm of the input image, (c) Tree 1 of the DTCWT transform on the logarithm image, (d) Tree 2 of the DTCWT transform on the logarithm image, (e) set the approximation and the highest two resolution scales of tree 1 to zero, (f) set the approximation and the highest two resolution scales of tree 2 to zero, (g) the output illumination invariant face generated by inverse DTCWT.

We give detailed steps of our new face recognition methods LOG-DTCWT as follows.

\section{Algorithm LOG-DTCWT or LOG-DWT}

Step 1. Initialization: $J=4$.

Step 2. Take the logarithm transform of the intensity image $I(x, y)$ as $(2)$.

Step 3. Perform forward DTCWT or DWT transform $\log I(x, y)$ for $J$ scales, to get $B=D T C W T(\log (I), J)$ or $B=D W T(\log (I), J)$.

Step 4. Set the approximation sub-band and the two highest frequency DTCWT coefficient sub-bands to zero values.

Step 5. Conduct inverse DTCWT/DWT to the output image from Step 4 in order to obtain face image $D$.

Step 6. Normalize $D$ so that it has zero mean and unit variance.

Step 7. Set $E=D^{k}$, where $k=0.69$ is a constant.

Step 8. Use CRC to classify the resulting face image to one of the known classes. 


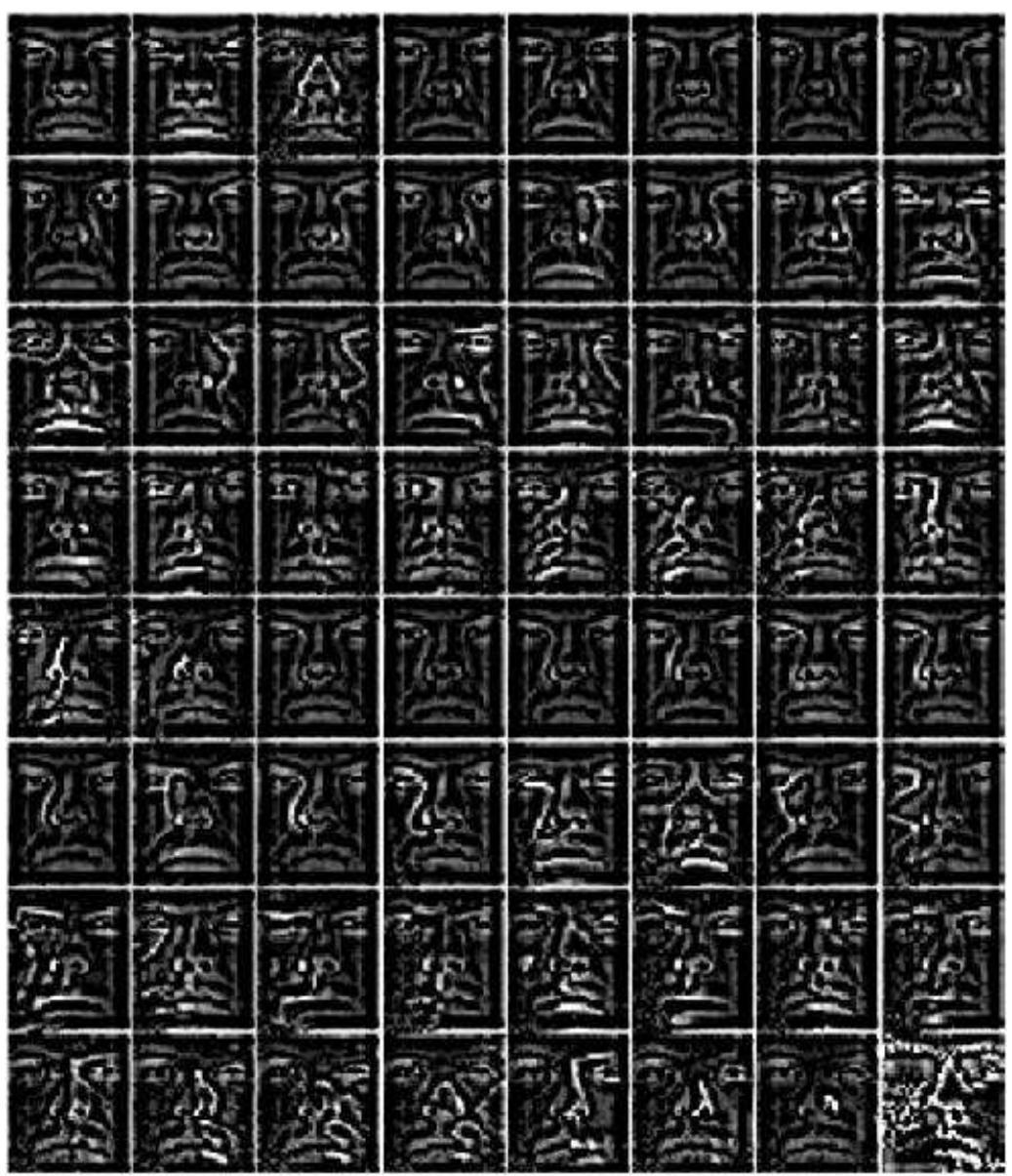

Fig. 6. A sample of the enhanced face images by our proposed method under different lighting condition of the extended Yale-B face database

Table 1. The five subsets of the Extended Yale Face Database, their corresponding angles, and the number of faces in each

\begin{tabular}{lcc} 
Subsets & Angles & Number of Faces \\
\hline Subset 1 & $1^{\circ} \leq$ angle $\leq 12^{\circ}$ & $7 \times 38$ \\
Subset 2 & $13^{\circ} \leq$ angle $\leq 25^{\circ}$ & $12 \times 38$ \\
Subset 3 & $26^{\circ} \leq$ angle $\leq 50^{\circ}$ & $12 \times 38$ \\
Subset 4 & $51^{\circ} \leq$ angle $\leq 77^{\circ}$ & $14 \times 38$ \\
Subset 5 & $78^{\circ} \leq$ angle & $19 \times 38$ \\
\hline
\end{tabular}

The computational complexity of our novel method can be summarized as follows. Suppose the input facial images have $M$ rows and $N$ columns. In addition, suppose the number of training images be $P$ and the number of testing images be $Q$. The logarithm transform is of linear complexity. The 2D DTCWT is linear as well. In addition, Step 6 in our new method has linear complexity because it calculates the power $(k=0.69)$ for every pixel. Therefore, the computational complexity of our proposed method is $O(P M N)$ for training, and $O(Q M N)$ for testing.
Note that this paper is an extension to our previous conference paper [31] published in the Proceedings of the International Conference on Pattern Recognition and Artificial Intelligence (ICPRAI), Montreal, QC, Canada, May 14-17, 2018.

\section{Data sets}

We conduct several experiments for face recognition on the Extended Yale Face Database B [4] and the CMUPIE illumination face database [27]. The Extended Yale B database [4] has face images of 38 subjects in 64 diverse lighting conditions: from normal to extremely badly illuminated. There is only one ideal image for each person. There are 2414 available images in total. We cropped and fixed the facial images to have $192 \times 168$ pixels. We take one well-lighted face image as the single reference and take all the rest available $2414-38=2376$ images as test samples. The faces are divided into 5 subsets according to angles between the light source direction and the camera axis.

The degree of variation gets higher from Subset 1 to Subset 5. Figure 4 shows the five subsets for one subject. Figure 5 displays the facial images in this database 


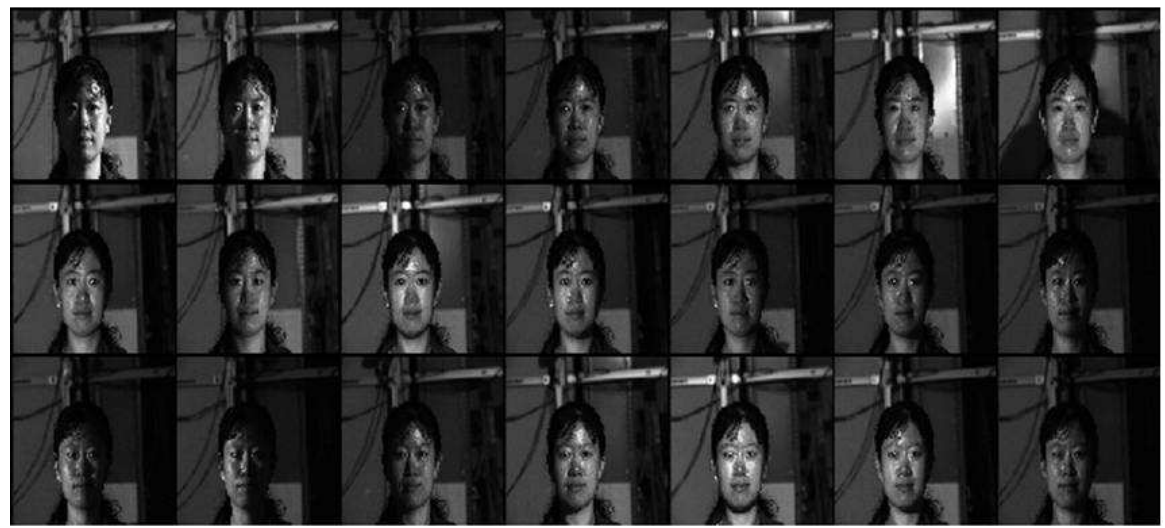

Fig. 7. A sample of the face images under different lighting condition of the CMU-PIE illumination face database

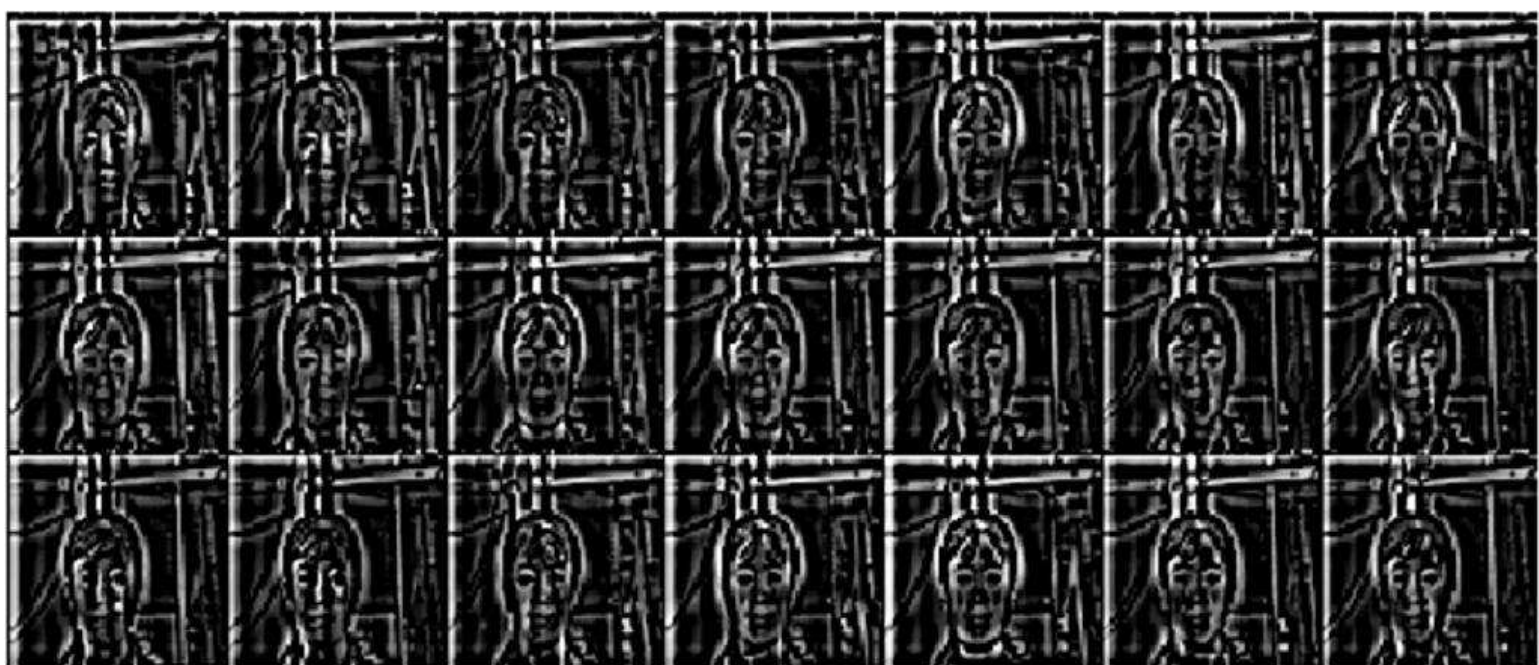

Fig. 8. A sample of the enhanced face images by our proposed method under different lighting condition of the CMU-PIE illumination face database

with diverse lighting conditions and Fig. 6 depicts the produced facial images by our proposed algorithm in this article. It is not difficult to see that our proposed method performs well in illumination invariant face recognition.

The CMU Pose, Illumination and Expression (PIE) database [27] has 41368 facial images for 68 persons. Each person has images captured for 13 poses and 43 illumination conditions. We only choose the images that focuses on illumination variations on light intensity and direction in frontal view. There are 68 persons in each 43 images yielding a total of 2924 images. Figure 7 displays a number of original facial images in this database while Fig. 8 shows the produced facial images in this database by using our LOG-DTCWT method.

For both databases, we choose only one frontally lit face image in each class for training and the remaining face images for testing. We transform every face image to the logarithm domain and normalize it to have zero mean and the pixel values fall in the range of $[0,255]$. We then perform DTCWT transform to these normalized face im- ages for $J=4$ scales and set the DTCWT coefficients to zero for the two highest frequency sub-bands and the approximation sub-band. An inverse DTCWT transform will generate the enhanced faces, which are approximately invariant to illumination changes and therefore good for face classification.

\section{Experimental results}

We tabulate the correct classification rates of our method and a number of existing methods in Table 2 for both face databases. The correct classification rate is defined as the percentage of faces that are recognized correctly regarding their true class labels. In this table, only LOG-DTCWT, LOG-DWT, DTCWT, DWT, and LOG are implemented by the authors. All other results are taken from Xie et al [28]. Our proposed algorithm yields higher classification rates in the Extended Yale Face Database B. For subset 1 of the Yale-B face database, our method has a classification rate of $93.4 \%$ 
Table 2. The correct classification rates (\%) of the proposed method LOG-DTCWT, and of the methods: LOG-DWT, LOG alone, DTCWT alone, DWT alone, Large and small scale [28], LOG-DCT [10], LTV [21], Local binary pattern [22], histogram equalization [23], and no features extraction (None). In this table, we copied the classification rates from [28] for LOG-DCT, LTV, Local binary pattern, histogram equalization, and no features extraction (None), the best results are boxed.

\begin{tabular}{|c|c|c|c|c|c|c|c|}
\hline \multirow[b]{2}{*}{ Methods } & \multirow[b]{2}{*}{ CMU-PIE } & \multicolumn{6}{|c|}{ Extended Yale Face Database B } \\
\hline & & Subset 1 & Subset 2 & Subset 3 & Subset 4 & Subset 5 & Average \\
\hline LOG-DTCWT & 100 & 93.4 & 100 & 91.1 & 88.5 & 87.3 & 92.0 \\
\hline LOG-DWT & 100 & 92.1 & 100 & 88.9 & 83.4 & 80.3 & 89.0 \\
\hline LOG & 99.78 & 98.25 & 99.12 & 67.89 & 30.76 & 26.04 & 64.41 \\
\hline DTCWT & 100 & 97.37 & \begin{tabular}{|l|}
100 \\
\end{tabular} & 47.89 & 10.69 & 4.29 & 52.05 \\
\hline DWT & 96.61 & 98.25 & 98.68 & 64.74 & 17.76 & 4.71 & 56.83 \\
\hline LSSF* & 99.9 & 100 & \begin{tabular}{|l|}
100 \\
\end{tabular} & 86.0 & 85.3 & 84.8 & 91.2 \\
\hline LOG-DCT (10) & 100 & 92.5 & 100 & 89.2 & 87.0 & 82.8 & 90.3 \\
\hline $\operatorname{LTV}(21)$ & 99.8 & 100 & 99.8 & 78.5 & 75.8 & 82.4 & 87.3 \\
\hline Local Binary Pattern (22) & 75.4 & 100 & 100 & 62.3 & 10.3 & 6.6 & 55.8 \\
\hline Histogram Equalization (23) & 42.2 & 99.1 & 94.7 & 43.2 & 12.2 & 15.4 & 52.9 \\
\hline None & 35.1 & 99.6 & 96.7 & 41.1 & 7.4 & 3.2 & 49.6 \\
\hline
\end{tabular}

LSSF* $^{*}$ Large and Small-Scale features [28]

Table 3. The correct classification rates (\%) of the proposed methods LOG-DTCWT, LOG-DWT, and LOG-DCT [16] for face images corrupted by Gaussian white noise. The ' 1 ' and ' 2 ' in the second column means that no noise is added to the reference face images, and noise is added to the reference images, respectively, the best results are boxed.

\begin{tabular}{|c|c|c|c|c|c|c|c|c|c|}
\hline \multirow[b]{2}{*}{ Databases } & \multirow[b]{2}{*}{ Methods } & \multicolumn{8}{|c|}{ Noise Standard Deviation (n) } \\
\hline & & 5 & 10 & 15 & 20 & 25 & 30 & 35 & 40 \\
\hline \multirow{6}{*}{$\begin{array}{l}\text { Extended } \\
\text { Yale Face } \\
\text { Database B }\end{array}$} & LOG-DTCWT 1 & 87.32 & 82.39 & \begin{tabular}{|l|}
79.61 \\
\end{tabular} & \begin{tabular}{|l|}
77.32 \\
\end{tabular} & 76.54 & \begin{tabular}{|l|}
75.70 \\
\end{tabular} & 74.47 & 73.71 \\
\hline & LOG-DWT 1 & 83.26 & 78.93 & 76.68 & 75.34 & 73.94 & 73.00 & 72.25 & 71.58 \\
\hline & LOG-DCT 1 & 87.28 & 82.28 & 78.85 & 77.17 & 75.85 & 74.59 & 73.94 & 73.53 \\
\hline & LOG-DTCWT 2 & 86.92 & 81.85 & 78.97 & \begin{tabular}{|l|}
76.61 \\
\end{tabular} & \begin{tabular}{|l|}
75.17 \\
\end{tabular} & \begin{tabular}{|l|}
73.89 \\
\end{tabular} & \begin{tabular}{|l|}
72.53 \\
\end{tabular} & 71.23 \\
\hline & LOG-DWT 2 & 82.61 & 77.21 & 74.09 & 71.35 & 68.76 & 66.75 & 64.64 & 61.52 \\
\hline & LOG-DCT 2 & 82.64 & 75.66 & 71.44 & 67.94 & 62.21 & 59.45 & 52.48 & 50.37 \\
\hline \multirow{6}{*}{ CMU-PIE } & LOG-DTCWT 1 & 100 & 100 & 100 & 100 & 100 & 100 & 100 & 100 \\
\hline & LOG-DWT 1 & 100 & 100 & 100 & 100 & 100 & 100 & 100 & 100 \\
\hline & LOG-DCT 1 & 100 & 100 & 100 & 100 & 100 & 100 & 100 & 100 \\
\hline & LOG-DTCWT 2 & 100 & 100 & 100 & 100 & 100 & 100 & \begin{tabular}{|l|}
99.91 \\
\end{tabular} & 99.91 \\
\hline & LOG-DWT 2 & 100 & 100 & 100 & 100 & 100 & 100 & \begin{tabular}{|l|}
99.91 \\
\end{tabular} & 99.81 \\
\hline & LOG-DCT 2 & 99.91 & 99.05 & 98.86 & 98.30 & 92.72 & 97.63 & 96.97 & 96.22 \\
\hline
\end{tabular}

whereas Large and Small-Scale features [28], LTV [11] and Local Binary Pattern [22] all obtained perfect classification rate $(100 \%)$. Xie et al [28] developed an illumination invariant face recognition technique on large and small-scale features for face recognition. They achieved $91.2 \%$ correct recognition rate for the Extended Yale database B. This recognition rate is higher than the recognition rates of all other methods except our method LOGDTCWT. Our proposed method LOG-DTCWT achieves $92.0 \%$ average classification rate, which is higher than all other methods compared in this paper. This shows the advantage of our proposed method for robust face recognition for the Extended Yale database B. For the CMU-PIE illumination face database, our proposed algorithm obtains $100 \%$ correct classification rate whereas other methods obtain equal or lower classification rates than our method. In summary, our proposed algorithm in this article is preferable for illumination invariant face recognition.

We tested our proposed method with LOG-DCT [10] for noisy faces. Figur 9 shows the noisy faces corrupted by Gaussian white noise with different noise standard devia- 


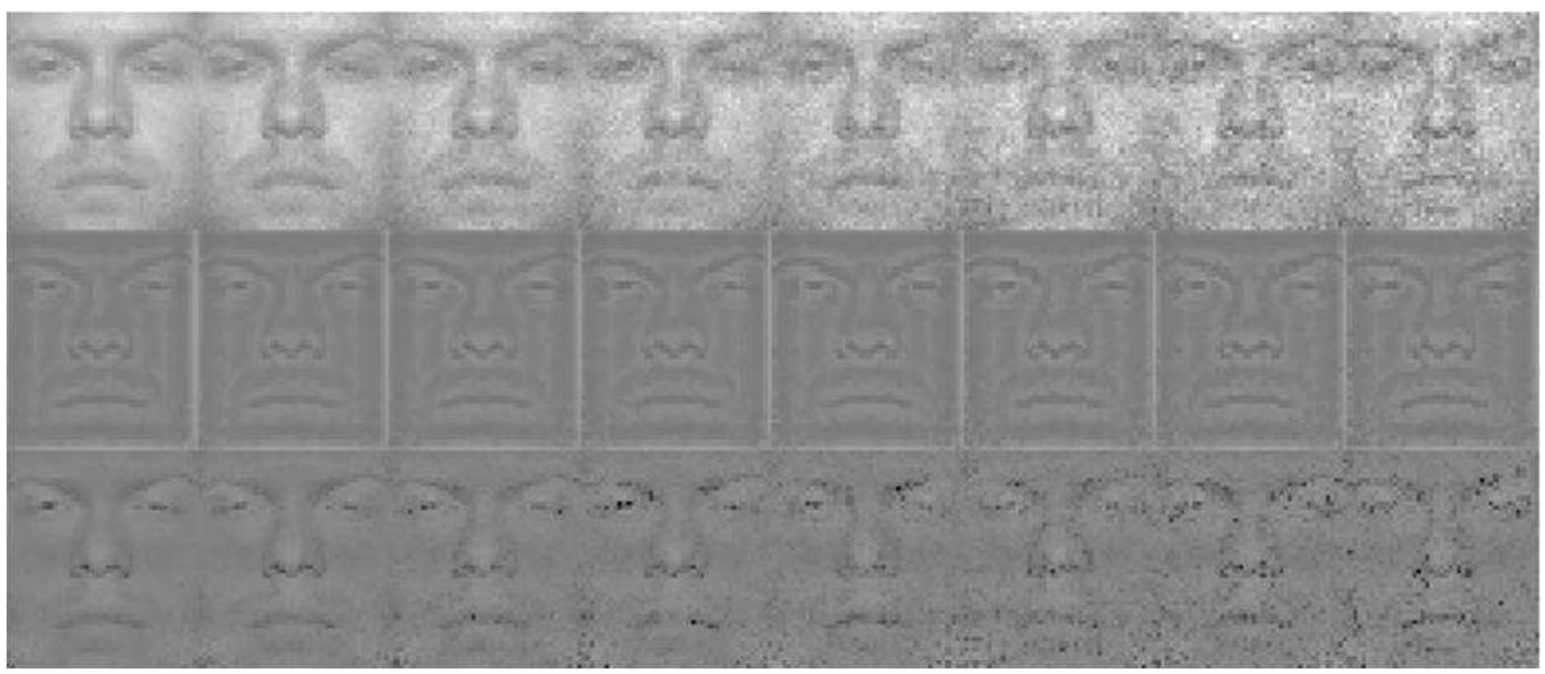

Fig. 9. First row: A list of face images corrupted by gaussian white noise with noise standard deviation $\sigma_{n}$ increases from 5 to 40 . Second row: the LOG-DTCWT faces proposed in this paper. Third row: the LOG-DCT faces [10]. It can be seen that our LOG-DTCWT faces do not change much with regard to noise levels

tion, the LOG-DTCWT faces proposed in this paper, the LOG-DWT faces, and the LOG-DCT faces [10]. As can be seen the LOG-DTCWT faces do not change much regarding noise levels. Table 3 show the correct classification rates $(\%)$ of the proposed method LOG-DTCWT, LOGDWT, and LOG-DCT [16] for face images corrupted by Gaussian white noise. The ' 1 ' and ' 2 ' in the second column means that no noise is added to the reference face images, and noise is added to the reference images, respectively. We see that our proposed method LOG-DTCWT improves upon the LOG-DWT and the LOG-DCT for all test cases.

\section{Conclusions}

In this article, we have developed a new algorithm for face recognition by extracting DTCWT faces in logarithm domain. Our algorithm is relatively invariant to illumination changes in the face images. The CRC is used as a classifier in our method. Our proposed algorithm is superior to existing algorithms in terms of correct recognition rates for the Extended Yale Face Database B. Our algorithm improves upon or is comparable to every algorithm for the CMU-PIE illumination face database. Our proposed algorithm in this article is more suitable for recognizing facial images with varying illumination and even for noisy environments.

The contributions of this paper can be described as follows. In our proposed algorithm, we perform logarithm transform to make dark regions brighter and we use DTCWT transform to generate illumination invariant face images. This combination of logarithm-DTCWT is new to our best knowledge. Our proposed method does not generate discontinuing region boundary as the method in [8], and it does not need to smooth the face image as Gradient faces [14]. In addition, our new method performs very well under noisy environment, but the LOG-DCT does not. Our method is easy to implement and for most test cases they yield higher recognition rates than a few existing methods compared in this paper.

Future research will be conducted for other tough topics in invariant face recognition such as differences in shift, pose and expression, etc. We may apply robust PCA [30] for illumination invariant face recognition as well.

\section{Acknowledgement}

The authors would like to thank the anonymous reviewers and the associate editor for their constructive suggestions and comments, which improve the quality of this paper.

\section{REFERENCES}

[1] M. Turk and A. Pentland, "Eigenfaces for Recognition", Journal of Cognitive Neuroscience vol. 3, 1991, pp. 71-86.

[2] M. Turk and A. Pentland, "Face Recognition using Eigenfaces", Proceedings of IEEE Conference on Computer Vision Pattern Recognition (CVPR), 3-6 June 1991, pp. 586-591.

[3] X. He, S. Yan, Y. Hu, P. Niyogi and H. J. Zhang, "Face Recognition using Laplacian Faces", IEEE Transactions on Pattern Analysis Machine Intelligence vol. 27, 2005, pp. 328-340.

[4] K. C. Lee, J. Ho and D. Kriegman, "Acquiring Linear Subspaces for Face Recognition under Variable Lighting", IEEE Transactions on Pattern Analysis Machine Intelligence vol. 27, 2005, pp. 684-698.

[5] K. Luu, M. Savvides, T. D. Bui, and C. Y.. Suen, "Compressed Submanifold Multifactor Analysis with Adaptive Factor Structures", Proceedings of International Conference on Pattern Recognition Japan, pp. 11-15, Nov 2012.

[6] L. H. Yang, T. D. Bui and C. Y. Suen, "Image Recognition based on Nonlinear Wavelet Approximation", International Journal of Wavelets Multiresolution Information Processing vol. 1, 2003, pp. 151-162. 
[7] J. Wright, A. Y. Yang, A. Ganesh, S. Sastry and Y. Ma, "Robust Face Recognition via Sparse Representation", IEEE Transactions on Pattern Analysis Machine Intelligence vol. 31, 2009, pp. 210-227.

[8] S. Du and R. K. Ward, "Adaptive Region-Based Image Enhancement Method for Robust Face Recogition under Variable Illumination Conditions", IEEE Transactions on Circuits Systems for Video Technology vol. 20, 2010, pp. 1165-1175.

[9] J. Ruiz-Pinales, J. J. Acosta-Reyes, A. Salazar-Garibay and R. Jaime-Rivas, "Shift Invariant Support Vector Machines Face Recognition System", World Academy of Science Engineering Technology vol. 16, 2008, pp. 947-951.

[10] W. Chen, M. Er and S. Wu, "Illumination compensation Normalization for Robust Face Recognition using Discrete Cosine Transform in Logarithm Domain", IEEE Trans, Syst, Man, Cybern. B, Cybern vol. 36, pp. 458-466.

[11] T. Chen, W. Yin, X. S. Zhou, D. Comaniciu, and T. S. Huang, "Total Variation Models for Variable Lighting Face Recognition", IEEE Transactions on Pattern Analysis Machine Intelligence vol. 28, 2006, pp. 1519-1524.

[12] T. Ahonen, A. Hadid, and M. Pietikainen, "Face Description with Local Binary Patterns: Application to Face Recognition", IEEE Transactions on Pattern Analysis Machine Intelligence vol. 28, 2006, pp. 2037-2041.

[13] C. Liu and D. Q. Dai, "Face Recognition using Dual-Tree Complex Wavelet Features", IEEE Transactions on Image Processing vol. 18, 2009, pp. 2593-2599.

[14] T. Zhang, Y. Y. Tang, B. Fang, Z. Shang, and X. Liu, "Face Recognition under Varying Illumination using Gradient Faces", IEEE Transactions on Image Processing vol. 18, 2009, pp. 2599-2606.

[15] Z. R. Lai, D. Q. Dai, C. X. Ren, and K. K. Huang, "Multiscale Logarithm Difference Edge Maps for Face Recognition Against Varying Lighting Conditions", IEEE Transactions on Image Processing vol. 24, 2015, pp. 1735-1747.

[16] J. H. Shah, M. Sharif, M. Raza, M. Murtaza, and S. Ur-rehman, "Robust Face Recognition Technique under Varying Illumination", Journal of Applied Research Technology vol. 13, 2015, pp. 97-105.

[17] I. W. Selesnick, R. G. Baraniuk, and N. G. Kingsburg, "The Dual-Tree Complex Wavelet Transform - a Coherent Framework for Multiscale Signal Image Processing", IEEE Signal Processing Magazine vol. 22, 2005, pp. 123-151.

[18] G. Y. Zhang, S. Y. Peng, and H. M. Li, "Combination of Dual-Tree Complex Wavelet SVM for Face Recognition", in Proc, Int. Conf. Machine Learning Cybernetics 12-15 July 2008, pp. 2815-2819.
[19] Y. H. Sun and M. H. Du, "Dt-CWT Feature Combined with ONPP for Face Recognition", in Computational Intelligence an Security, Berlin/Heidelberg Germany: Springer 2007, pp. 1058-1067.

[20] Z. M. Huang, X. H. Zhang, and D. Yang, "Face Recognition based on Dual-Tree Complex Wavelet Feature", Journal of Computer Application vol. 27, 2007, pp. 1135-1137.

[21] N. G. Kingsbury, "Complex Wavelets for Shift Invariant Analysis Filtering of Signals", Journal of Applied Computational Harmonic Analysis vol. 10, pp. 234-253.

$22]$ B. K. P. Horn, "Robot Vision", Cambridge, MA: MIT Press, 1997.

[23] L. Zhang, M. Yang, and X. Feng, "Sparse Representation or Collaborative Representation: which Helps Face Recognition?", IEEE International Conference on Computer Vision, 2011, pp. 471-478.

[24] G. Y. Chen, T. D. Bui, and A. Krzyzak, "Invariant Pattern Recognition using Radon, dual-tree complex wavelet and Fourier transforms,", Pattern Recognition vol. 42, no. 9, pp. 2013-2019 2009.

[25] G. Y. Chen and W. P. Zhu, "Signal Denoising using Neighbouring Dual-Tree Complex Wavelet Coefficients", IET Signal Processing vol. 6, 2012, pp. 143-147.

[26] G. Y. Chen and W. F. Xie, "Contour-based Feature Extraction using Dual-Tree Complex Wavelets", International Journal of Pattern Recognition Artificial Intelligence vol. 21, 2007, pp. 1233-1245.

[27] T. Sim, S. Baker, and M. Bsat, "The CMU Pose, Illumination", IEEE Transactions on Pattern Analysis Machine Intelligence vol. 25, 2003, pp. 1615-1618.

[28] X. Xie, W. Zheng, J. Lai, P. C. Yuen, and C. Y. Suen, "Normalization of Face Illumination based on Large Small-Scale Features", IEEE Transaction on Image Processing vol. 20, 2011 pp. 1807-1821.

[29] R. C. Gonzalez and R. E. Woods, "Digital Image Processing" 2008, Third Edition.

[30] K. G. Quach, C. N. Duong, K. Luu, and T. D. Bui, "Non-Convex Online Robust PCA: Enhance Sparsity via $l_{p}$-Norm Minimization", Computer Vision Image Understanding vol. 158, 2017, pp. 126-140.

31] G. Y. Chen, T. D. Bui and A. Krzyzak, "Illumination Invariant Face Recognition using Dual-Tree Complex Wavelet Transform in Logarithm Domain", Proceedings of the International Conference on Pattern Recognition Artificial Intelligence (ICPRAI) Montreal, QC, Canada, May 14-17, 2018.

Received 11 April 2018 